\title{
Mechanistic Insight into the Carbon Dots: Protonation induced Photoluminescence
}

Syamantak K, Navneet CV, Prashant G, Sanjhal J, Souvik G and Chayan K Nandi*

School of Basic Sciences, Indian Institute of Technology, Mandi, Himachal Pradesh, India

\begin{abstract}
Although several theories have been proposed, the mechanism of complex photoluminescence in carbon dots (CNDs) is a central quest till date. This report presents $\mathrm{pH}$ dependent steady state and time resolved spectroscopy study which identifies a possible origin of the complex photoluminescence in CNDs. The multiple emissive species created by the excited state protonation-deprotonation reaction at certain $\mathrm{pH}$ gives rise to inhomogeneous broadening and consequently excitation dependent multicolour emission. The origin of the excited state dynamics is attributed to the significant change of the proton dissociation between ground and excited state. We present a new model on protonation dynamics and show how it affects the emissive states in CNDs.
\end{abstract}

Keywords: Carbon dots; $\mathrm{pH}$ dependence; Excited state protonation, time resolved fluorescence, origin of photoluminescence

\section{Introduction}

In spite of a number of theories including quantum confinement, [1] surface trap emission, [2,3] variable oxidation states, [4] aggregation induced emission, [5] presence of multichromophoric group, [6] crystalline and amorphous carbon cluster $[7,8]$ and presence of molecular fluorophores, [9] which have been proposed by both experimental and theoretical studies, [10-12] a clear understanding of the mechanism of photoluminescence of carbon nanodots (CNDs) is lacking till date. Unlike semiconductor quantum dots, one of the major issues in CNDs is the use of different precursor molecules to synthesize it. Depending on the synthesis condition, different types of surface functional groups $(\mathrm{COOH}, \mathrm{OH}, \mathrm{C}=\mathrm{O}$ and $\mathrm{NH}_{2}$ ) in $\mathrm{CNDs}$ can be present. Although most CNDs are found to display considerable similarities in their optical properties, different mechanisms of their photoluminescence have been proposed. However, it is understood that the functional groups play a pivotal role and can act differently in different CNDs.

Excitation dependent red-shifting of their emission spectra remained another key topic of debate. This phenomenon too, is strongly influenced by the surface chemistry of CNDs. For instance, recent reports suggested the existence of a critical $\mathrm{pH}$ below which the excitation dependent red shifted multicolour emission is observed $[13,14]$. However, it vanishes above the critical pH. The similar phenomena was also observed in a recent report in graphene oxide (GO) [15] functionalized with $\mathrm{COOH}, \mathrm{OH}$ and $\mathrm{NH}_{2}$, which showed three pKa values at 4.3, 6.6 and 9.8. These were designated as dissociation of carboxylic acid in presence of hydroxyl group, isolated carboxylic acid and phenolic $\mathrm{OH}$ group. Further, the excited state proton transfer was observed, leading to spectral migration of GO. The above observations were described mainly by the effect of $\mathrm{pH}$ on the carboxylic acids present on the surface of CNDs or GO. All these studies show a spectral redshift in absorption and emission with decreasing $\mathrm{pH}$ of the solution. Interestingly, another recent study reports just the opposite trends of the spectral changes, where spectral blue shifts in both the absorption and emission spectra were observed [16]. The pKa value obtained as 7.9, which supports the phenol-phenolate equilibrium responsible for the spectral changes, although large number of carboxylic acid was present in the CNDs. This led us to a common hypothesis that the type and the predominance of functional groups and their $\mathrm{pH}$ dependent protonation-deprotonation kinetics either in ground or excited states determine the fate of spectral changes in CNDs [17].

\section{Materials and Methods}

\section{Materials}

All Glass wares were washed with aqua regia $\left(3 \mathrm{HCl}: 1 \mathrm{HNO}_{3}\right)$, followed by rinsing several times with double distilled water. Potato dextrose agar was purchased from Sigma Aldrich. $\mathrm{NaOH}$ purchased from Merck chemicals. Double distilled $18.3 \mathrm{~m} \Omega$ deionized (DI) water (Elga Purelab Ultra) was used throughout for the preparation of solutions.

\section{Synthesis of CNDs}

For the synthesis of CNDs, $2 \mathrm{gm}$ of PDA broth was dissolved in $2 \mathrm{ml}$ of distilled water and $500 \mu \mathrm{L}$ of $\mathrm{H}_{3} \mathrm{PO}_{4}$ was added onto it. The mixture was heated at up to $180^{\circ} \mathrm{C}$ until the solution became thick. After this 1 $\mathrm{ml}$ of water was added and the heating was continued until the solution became thick gain. The last step was repeated thrice. The resulting material (chocolate brown ash) is diluted up to $100 \mathrm{ml}$ in distilled water and the solution was filtered by Whatman filter paper. The solution was ultracentrifuged (sorvall Lynx 6000, thermo scientific) for two times successively at $23000 \mathrm{rpm}$. Each run was carried out for 30 minutes. The pellets were removed each time and the final supernatant was collected. This solution was dialyzed for 2 days using 3-4 kD membrane against water.

\section{UV-Vis absorption spectroscopy}

The UV-Vis absorption spectra were recorded using Shimadzu UVVis 2450 spectrophotometer. The spectra were collected using a quartz cuvette of $10 \mathrm{~mm}$ path length and volume $1 \mathrm{ml}$. All the measurements were repeated at least three times.

*Corresponding author: Chayan K Nandi, School of Basic Sciences, Indian Institute of Technology, Mandi, Himachal Pradesh, India, Tel: 01905267047; E-mail: chayan@iitmandi.ac.in

Received April 06, 2018; Accepted April 30, 2018; Published May 10, 2018

Citation: Syamantak K, Navneet CV, Prashant G, Sanjhal J, Souvik G, et al. (2018) Mechanistic Insight into the Carbon Dots: Protonation induced Photoluminescence. J Material Sci Eng 7: 448. doi: 10.4172/2169-0022.1000448

Copyright: (c) 2018 Syamantak K, et al. This is an open-access article distributed under the terms of the Creative Commons Attribution License, which permits unrestricted use, distribution, and reproduction in any medium, provided the original author and source are credited. 


\section{Transmission electron microscope (TEM)}

The particle size and dispersity of the synthesized CNDs were checked using a TECNAI G2 $200 \mathrm{kV}$ TEM (FEI, Electron Optics) electron microscope with $200 \mathrm{kV}$ input voltage. TEM grids were prepared by placing $5 \mu \mathrm{L}$ diluted and well sonicated sample solutions on a carbon coated copper grid and evaporated the solution at room temperature completely. Precautions were taken to avoid contamination from various sources like dust particles and glasswares.

\section{Fourier transform infrared spectroscopy (FTIR)}

FTIR spectra of CNDs were measured using an Agilent FTIR spectrophotometer equipped with a horizontal attenuated total reflectance (ATR) accessory containing a zinc selenide crystal and operating at $4 \mathrm{~cm}^{-1}$ resolution. The use of the spectral subtraction provided reliable and reproducible results.

\section{Atomic force microscopy (AFM)}

AFM analysis of the synthesized CNDs for particle size determination was carried out using a Digital Instruments Bruker AFM. Standard Veeco tapping mode silicon probes were used for scanning the samples. Typically, aqueous suspensions of CNDs samples were dried on silicon substrate for 3 hours. Once dried, samples were placed on the AFM stage and scanned. Pertinent scanning parameters were as follows: Resonant frequency (probe): $60-80 \mathrm{kHz}$; Tip velocity for all measurements are: $(4 \mu \mathrm{m} / \mathrm{s}$ for $2 \mu \mathrm{m}),(15 \mu \mathrm{m} / \mathrm{s}$ for $5 \mu \mathrm{m}),(30 \mu \mathrm{m} / \mathrm{s}$ for $10 \mu \mathrm{m})$. Aspect ratio: 1:1; Resolution: 512 samples/line, 256 lines.

\section{X-ray photoelectron spectroscopy (XPS)}

X-ray Photo-Electron Spectroscopy (XPS) with Auger Electron Spectroscopy (AES) module PHI 5000 Versa Prob II, FEI Inc. and C60 sputter gun have been used for characterization and scanning

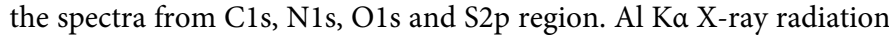
was used as the source for excitation $(1486.8 \mathrm{eV}, 500 \mathrm{~mm})$. Samples were loaded on copper strips, and surface adherence done by double sided adhesive tape. CNDs samples were prepared by concentrating the solution by centrifugation at $23000 \mathrm{rpm}$, followed by drying. Thus obtained CNDs samples were used for recording XPS spectra.

\section{Steady state and time resolved fluorescence spectroscopy}

Steady state fluorescence was measured using Horiba Fluorolog-3 Spectrofluorometer. All the experiments were performed at room temperature. The fluorescence was measured in $1 \mathrm{ml}$ quartz cuvette. The fluorescence lifetime and time resolved emission spectra (TRES) were measured using Horiba scientific Delta Flex TCSPC system with Pulsed LED Sources. Ludox has been used as an IRF for de-convolution of the spectral value. The photon decays in different channels were fitted tri-exponentially with a chi-squared value $<1.2$ in order to calculate fluorescence lifetime. TRES was plotted by taking 50 slices of the $3 \mathrm{D}$ plot at 13 channel ( $0.35 \mathrm{~ns})$ interval. Approximately $3 \mathrm{ml}$ CNDs solution has been used for all measurements.

\section{Result and Discussion \\ Properties of CNDs at different $\mathrm{pH}$}

The CNDs was synthesized from potato dextrose broth (PDB) and the detail synthesis protocol and complete characterization of the CNDs could be found in the supporting information 17 (Supplementary Figure S1). The FTIR data supports the presence of $\mathrm{COOH}$ and $\mathrm{OH}$ functional groups on the surface of CNDs. Figure 1a illustrate optical properties of CNDs at two different $\mathrm{pH}$ conditions. UV-Vis absorption spectra (dotted lines) show a single maximum at $280 \mathrm{~nm}$ both at $\mathrm{pH}$ 3 and 10, and it doesn't change with change in $\mathrm{pH}$ of the solution. Interestingly, the fluorescence spectra (solid lines, Figure 1a) of CNDs show strong dependence on the $\mathrm{pH}$ of the solution, indicating to an excited state phenomenon. At higher $\mathrm{pH}$, the $\mathrm{CNDs}$ shows fluorescence spectrum with a single emission maxima at $360 \mathrm{~nm}$. But at lower $\mathrm{pH}$ $(\sim \mathrm{pH} 3)$, a secondary highly red shifted emission maximum arises at $465 \mathrm{~nm}$. It also suggests that there exist a critical $\mathrm{pH}$ below which the protonated $(\mathrm{COOH}$ and $\mathrm{OH})$ species emit and above the critical $\mathrm{pH}$ the anionic species (COO- and $\mathrm{OH}-$ ) emission occurs. Interestingly, the $\mathrm{pH}$ dependent zeta potential strongly supports the above conclusion (Figure $1 \mathrm{~b}$ ). The CNDs shows a negative zeta potential up to $-20 \mathrm{mV}$ at $\mathrm{pH}>10$ and it increases gradually with decrease in $\mathrm{pH}$. At $\mathrm{pH} 3$ it rises to zero and shows positive zeta potential value at lower $\mathrm{pH}$ (below 3 ). Besides, zeta potential analysis shows that the CNDs has an isoelectric point $\sim 4$ and a possible $\mathrm{pKa} \sim 2-4$. Therefore, for simplicity, we choose specifically two $\mathrm{pH}$ conditions for further comparative studies: $\mathrm{pH}$ 3 , where the zeta potential is maximum $(+1.9 \pm 0.3 \mathrm{mV}$, completely protonated), and $\mathrm{pH} 10$, where the zeta potential is minimum (-18 \pm 2. $\mathrm{mV}$, completely deprotonated). Considering the reported $\mathrm{pKa}$ value of 4.3 for $\mathrm{COOH}$ in the vicinity of $\mathrm{OH}$ in $\mathrm{GO}$, we suggest that $\mathrm{COOH}$ and $\mathrm{COO}$ - equilibrium controls the fluorescence process [15]. In contrast, the evidence of spectral blue shift with lowering $\mathrm{pH}$ in both a

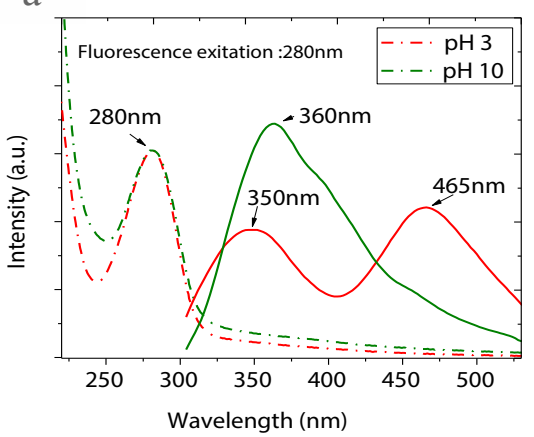

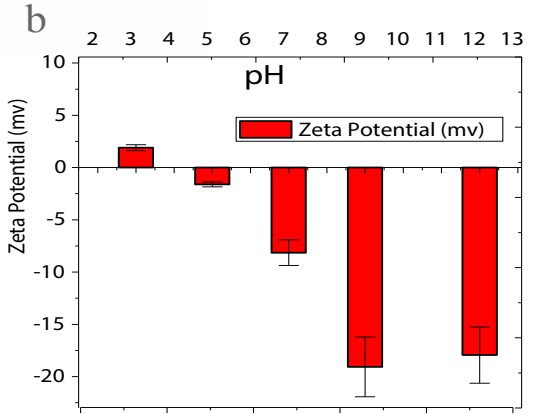

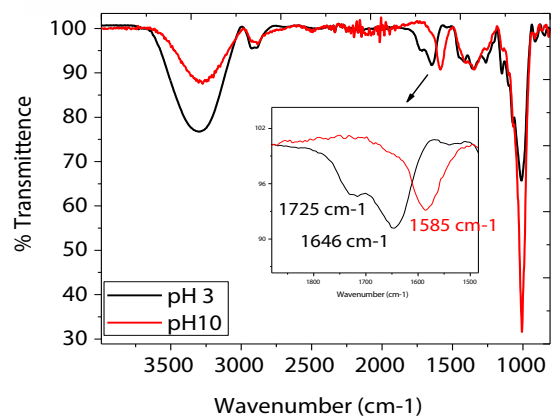

Figure 1: Properties of CNDs at different $\mathrm{pH}$ conditions. (a) Absorption spectra (dotted lines) show same absorption maxima at $280 \mathrm{~nm}$ unaltered at two $\mathrm{pH}$. The emission spectra, which were taken with $280 \mathrm{~nm}$ excitation, show two $(350 \mathrm{~nm}, 465 \mathrm{~nm})$ emission maxima at $\mathrm{pH}=3$ and a single maxima $(360 \mathrm{~nm})$ at $\mathrm{pH}=10$. (b) Zeta potential analysis shows decreasing value at lower $\mathrm{pH}$ with an isoelectric point near $\mathrm{pH} 4$ (c) FTIR spectra at pH 3 and pH 10 show carboxylic and carboxylate stretching vibrations respectively. 
Citation: Syamantak K, Navneet CV, Prashant G, Sanjhal J, Souvik G, et al. (2018) Mechanistic Insight into the Carbon Dots: Protonation induced Photoluminescence. J Material Sci Eng 7: 448. doi: 10.4172/2169-0022.1000448

the absorption and emission spectra and a measured pKa value of 7.9 corresponding to phenol-phenolate equilibrium (responsible for CNDs fluorescence) is reported recently [16]. As a result, it is quite obvious that the type and the predominance of functional group $(\mathrm{COOH}, \mathrm{OH}$ or $\mathrm{NH}_{2}$ etc.) determines the protonated deprotonated dissociation kinetics and hence the spectral changes. The major role of $\mathrm{COOH}$ and COO- equilibrium is also evident from the FTIR data (Figure 1c). In acidic condition as the carboxyl group remains protonated, FTIR shows a strong broad peak with maximum at $3300 \mathrm{~cm}^{-1}$ corresponding to the $\mathrm{O}-\mathrm{H}$ stretching vibrations and other peaks corresponding to $\mathrm{C}=\mathrm{O}$ stretching of undissociated carboxylic acid at $1725 \mathrm{~cm}^{-1}$ and $\mathrm{C}=\mathrm{C}$ stretching at $1646 \mathrm{~cm}^{-1}$. At higher $\mathrm{pH}$ the peak at $1725 \mathrm{~cm}^{-1}$ due to $\mathrm{C}=\mathrm{O}$ stretching of undissociated carboxylic acid disappears and gives rise to a new peak at $1585 \mathrm{~cm}^{-1}$ that is attributed to the stretching of the dissociated and conjugated carboxylate groups.

\section{Time resolved fluorescence studies}

To elucidate the excited state dynamics more clearly, we measured time resolved emission spectra (TRES) [18] and time resolved area normalised emission spectra (TRANES) [19]. The TRES is a powerful technique to identify multiple emissive excited states. Time resolved area normalized emission spectra (TRANES) is a unique analysis of TRES where the number of emissive species can be identified accurately $[18,19]$. The ionic species which arises at lower $\mathrm{pH}$ shows a slow decaying process in the TRES (Figure $2 \mathrm{a}$ and $2 \mathrm{~b}$ ). Further, the two energy states $(\sim 350 \mathrm{~nm}$ and $\sim 450 \mathrm{~nm})$ decays at different time scales, indicating their different excited state lifetimes (Figure $2 \mathrm{c}$ and $2 \mathrm{~d}$ ). At $\mathrm{pH} 3$, the lifetime at $450 \mathrm{~nm}$ was found to be $8.3 \mathrm{~ns}$, which is fivefold higher than $350 \mathrm{~nm}(\sim 1.6 \mathrm{~ns})$. However, the lifetimes of $450 \mathrm{~nm}$ and 350 $\mathrm{nm}$ states are nearly equal ( $2 \mathrm{~ns})$ at $\mathrm{pH} 10$. It is interesting to note that the lifetime at $460 \mathrm{~nm}$ gradually increases and lifetime at $350 \mathrm{~nm}$ state slightly deceases when the $\mathrm{pH}$ is gradually lowered (Supplementary Figure S2). This anti-correlation points towards a $\mathrm{pH}$ induced interconversion kinetics, where one state depopulates and the other populates. TRANES shows fluorescence emission form two excited states $(\sim 340 \mathrm{~nm}$ and $\sim 450 \mathrm{~nm})$ at $\mathrm{pH}=3$ at nanosecond time scale (Figure 2e). An isoemissive point in TRANES around $400 \mathrm{~nm}$ is a clear evidence of the presence of two emissive species [20,21]. In contrast, the TRANES at higher $\mathrm{pH}$ (Figure 2f) remains unaltered, resulting in no observable spectral migration up to $30 \mathrm{~ns}$. As the secondary species is only formed at low $\mathrm{pH}$ conditions, the phenomenon can be attributed to a protonation reaction of a $\mathrm{COO}$ - functional group. a

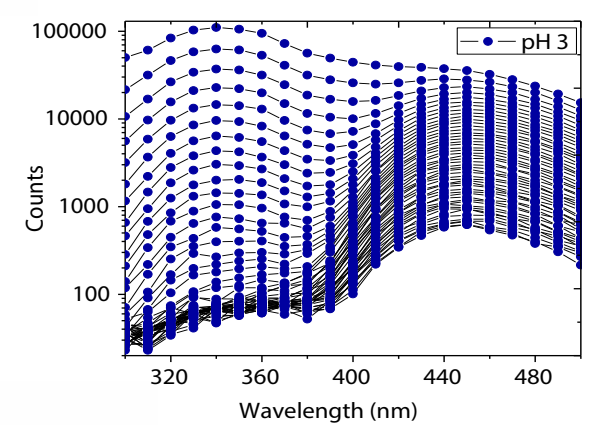

$\mathrm{c}$

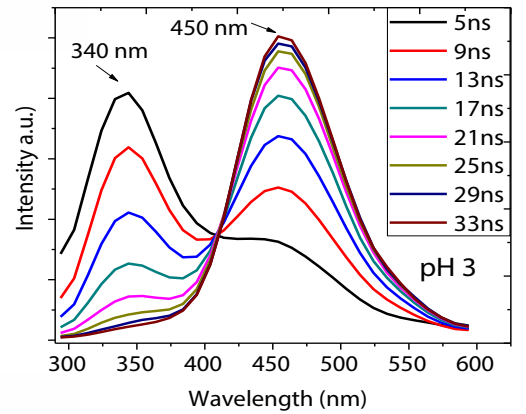

e

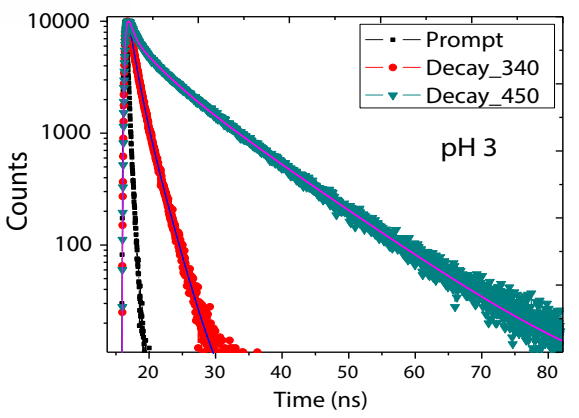

b

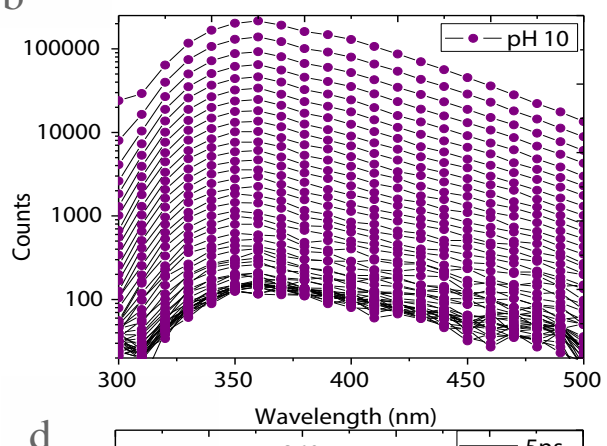

d
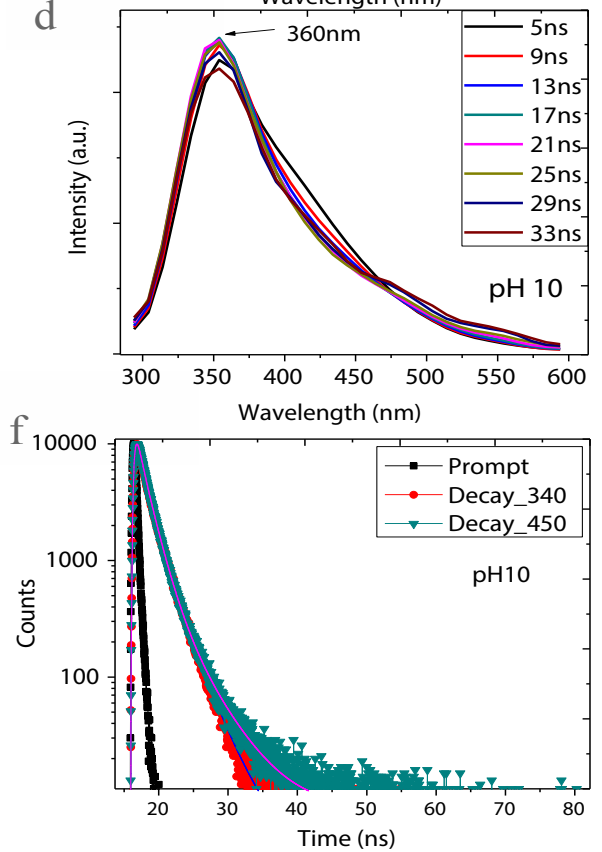

Figure 2: Time resolved fluorescence study of CNDs. ( $a$ and b) TRES, ( $c$ and d) TRANES and (e and f) fluorescence decay curve at low and high pH respectively. A spectral migration at $\mathrm{pH}=3$ and an unchanged spectra at $\mathrm{pH}=10$ is observed in TRES and TRANES between $5 \mathrm{~ns}$ to $33 \mathrm{~ns}$. The fluorescence lifetime at 450 $\mathrm{nm}$ is nearly fivefold higher than the lifetime at $350 \mathrm{~nm}$. 
The observed large change in lifetime can be understood from the model of excited state reaction presented in Figure 3a, where the unreacted excited state $(\mathrm{U})$ relaxes to a lower energy reacted excited state (R). In absence of any excited state reaction, the state returns to the ground state with a rate $k R+k N R$. The reaction rate $(k 1)$ is dependent on the hydrogen ion concentration $(\mathrm{pH})$ and there exists a critical $\mathrm{pH}$, above which the fluorescence occurs only from the $U$ state and below which it occurs from both. As the $\mathrm{U}$ state converts to the $\mathrm{R}$ state with a rate $k 1$, the $U$ state depopulates with an overall rate of $(k R+k N R)$ $+k 1$ assuming irreversibility. So the reaction rate or the time scale of protonation can be roughly estimated (assuming first order kinetics) by solving the simultaneous equations $\frac{1}{\left(k_{R}+K_{N R}\right)+K_{1}}=1.6 n s$ and $\frac{1}{\left(k_{R}+K_{N R}\right)}=2 n s$. The rate of protonation $(k 1)$ is estimated to be approximately $1.25 \times 108 \mathrm{~s}^{-1}$. The inverse of $k 1$ gives an average time scale of $8 \mathrm{~ns}\left(\tau_{1}=1 / k 1\right)$ for the protonation reaction. The time scale of protonation can also be estimated from Figure $2 \mathrm{c}$ where the longer wavelength component $(465 \mathrm{~nm})$ grows significantly up to $30 \mathrm{~ns}$. Figure $3 \mathrm{~b}$ shows time dependent intensity plot of the $\mathrm{U}$ and $\mathrm{R}$ states as observed in Figure 2c. Single exponential fitting of each curve returns time constants $\tau \simeq 12 \mathrm{~ns}$, which is in the same order of 1 / $k 1$. The single exponential fit clearly indicates a first order reaction kinetics, validating our previous assumption. So these time-resolved data clearly suggest that the excited state reaction takes place in nanosecond time scale. Now, we try to correlate excited state protonation to the possible origin of photoluminescence of CNDs. It is well know that the excited state protonation and deprotonation is governed by the change of electron density of the molecule upon photo-excitation. This change in electron density, directly influences the dissociation rate or dissociation constant ( $\mathrm{pKa})$ in the excited state. For, instance, if the $\mathrm{pKa}$ value decreases upon excitation $\left(\mathrm{pKa}^{*}<\mathrm{pKa}\right)$, an excited state deprotonation is expected. Naphthol is a well-studied [22] example of this case, where pKa value drops from 9.2 to 2 upon excitation. This leads to deprotonation of hydroxyl group in the excited state. Conversely, excited state protonation is observed in case of $\mathrm{pKa}^{*}>\mathrm{pKa}$. Examples include a number of carboxylic acids like benzoic acid where pKa value increases from 4.2 to 9.5 upon photo-excitation. Therefore, CNDs with carboxyl functionalities can also have the similar effect. It is evident in the current study that the spectral migration at $\mathrm{pH} 3-5$ occurs due to $\mathrm{pKa}^{\star}>\mathrm{pKa}$ of carboxylic acid groups. The spectral shift which occurs due the excited state relaxation can be used to calculate this change in pKa value by using Foster cycle [23].

$$
\Delta p K_{A}=p K_{A}-p K_{A}^{*}=\frac{E_{H A}-E_{A^{-}}^{-}}{2.3 R T}
$$

a

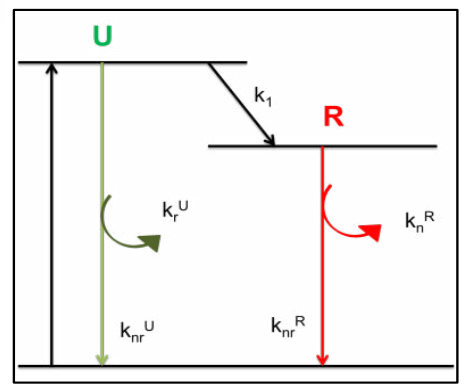

Where, $\mathrm{R}$ is the gas constant and $\mathrm{T}$ is the temperature $\left({ }^{\circ} \mathrm{K}\right)$. The energies of the protonated form (EHA) and dissociated form (EA-) is estimated form the average of the absorption $\left\langle\nu_{\mathrm{A}}\right\rangle$ and emission $\left\langle\nu_{\mathrm{F}}\right\rangle$ maxima of each species:

$$
E_{i}=N h c \frac{<v_{A}>+<v_{F}>}{2}
$$

Where $v$ is wavenumber in $\mathrm{cm}^{-1}, \mathrm{~h}$ is Planck constant, $\mathrm{N}$ is Avogadro's number and $\mathrm{c}$ is the speed of light. We calculated the $\Delta \mathrm{pKA}$ value to be $\sim-6.5$ using the above equation which consequently gives a value of $\mathrm{pKa}^{*}=\sim 8-10$. This represents a distinct increase of dissociation constant upon photo-excitation like benzoic acid and other carboxylic acids. With this evidence, we conclude that the fluorescence of $\mathrm{pH}$ sensitive CNDs originate mainly from the two ionic species $(\mathrm{COOH}$ and $\mathrm{COO}-$ ).

Now it could be argued whether the results obtained in this study are very specific to the CNDs obtained from $\mathrm{PDB}$ or similar precursors. However, we would like to emphasise here that this model could be pivotal to understand fundamental problem of CNDs system, in general. For instance, when we studied N-doped [13] and S-doped [14] CNDs, we found similar $\mathrm{pH}$ dependent exited state dynamics (Supplementary Figures S3 and S4). An isoemissive point is observed in every case, indicating the presence of two emissive species. Thus CNDs from $\mathrm{PDB}$ provides a unique opportunity to elucidate the excited state relaxations, which might be present but obscure in many other CNDs systems. It is also crucial to observe that our approach is not limited to CNDs with $\mathrm{COOH}$ functional groups. As already mentioned earlier, both protonation or de-protonation may occur depending on the $\left(\mathrm{pKa}-\mathrm{pKa}^{*}\right)$ value. Therefore $\mathrm{CNDs}$ with $\mathrm{OH}, \mathrm{NH}_{2}$ or any other protic functional group can be analysed similarly to elucidate their excited state dynamics.

\section{Relation with excitation dependent emission}

At this point, it is crucial to note that excitation dependent emission can also arise from the presence of two emissive states as described schematically in Scheme 1. If the steady state emission spectrum is considered as an overlap of two individual Gaussian functions, it can be understood from the scheme that, the intensity and the peak position of the emissive spectra is determined by the relative population of the protonated and deprotonated species in an additive manner (Scheme 1a). As the relative population changes with time, a migration of the integrated (steady state) spectra is observed, which manifest as excitation dependent red shift in the steady state fluorescence (Scheme 1b). Moreover, as the excited state depopulates with time, states that

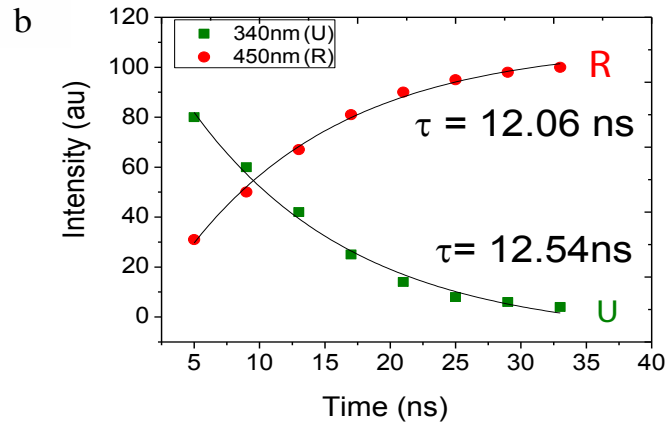

Figure 3: The two-state model. (a) Jablonski diagram for an irreversible excited state reaction. $U$ is an unreacted deprotonated state and $R$ is a reacted protonated state. (b) Time dependent fluorescence intensity plot of the $U$ and $R$ state as observed in TRANES at pH 3 . Single exponential fitting of each curve returns time constants $\sim 12 \mathrm{~ns}$, indicating a first order inter-conversion reaction. 
Citation: Syamantak K, Navneet CV, Prashant G, Sanjhal J, Souvik G, et al. (2018) Mechanistic Insight into the Carbon Dots: Protonation induced Photoluminescence. J Material Sci Eng 7: 448. doi: 10.4172/2169-0022.1000448
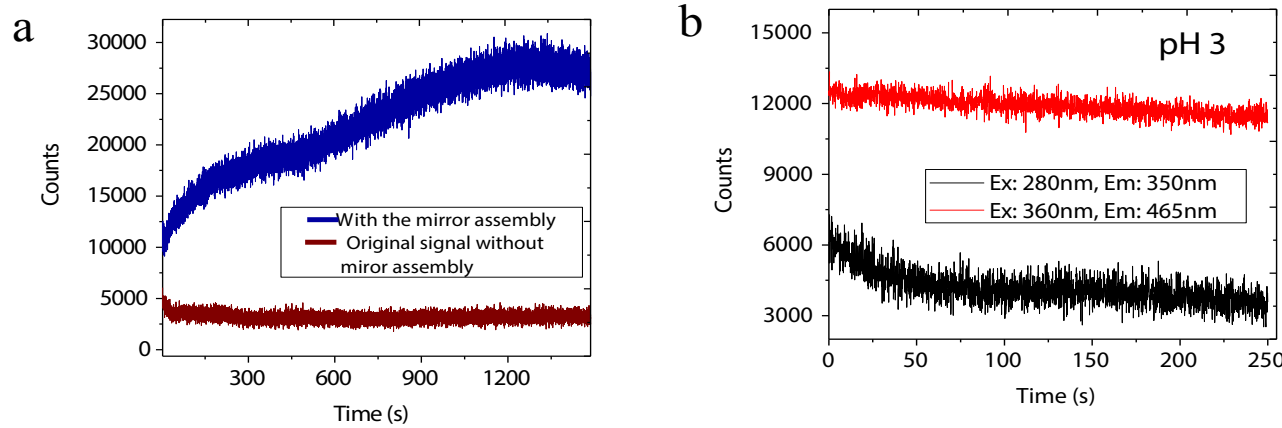

Figure 4: Amplified spontaneous emission (ASE) from CNDs. (a) ASE is observed only with the added mirror assembly at lower pH when the sample was excited at $280 \mathrm{~nm}$ and emission was collected at $465 \mathrm{~nm}$. (b) Control experiment shows that the other combinations of excitation and emission don't give rise to ASE, even with the mirror assembly at lower $\mathrm{pH}$.

$\mathrm{a}$

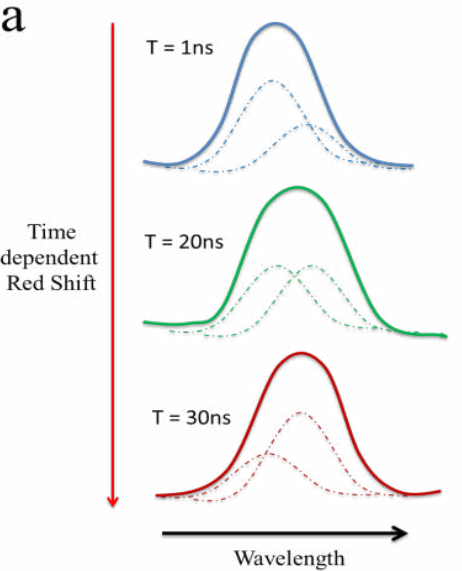

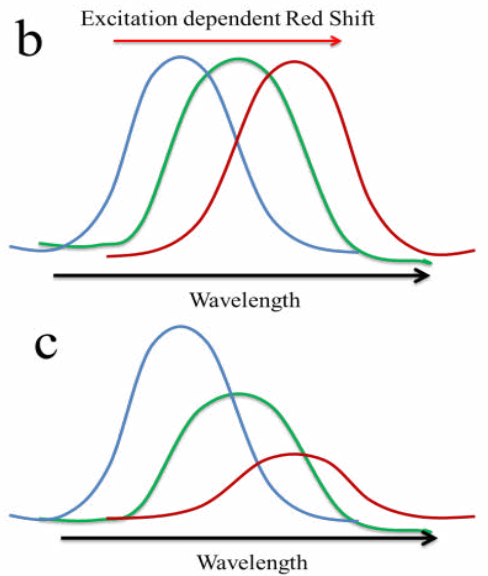

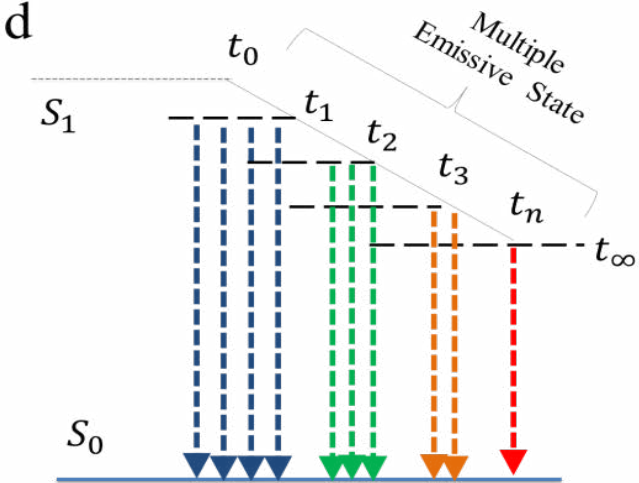

Decreasing Population

Scheme 1: Origin of excitation dependence emission. (a) The deprotonated and protonated species has different emission wavelength and an inter-conversion between them results different relative populations of the two emissive species at different time (Blue, green and red colour). (b)This gives rise to excitation dependent red shift in the steady state spectra. (c) Excited state depopulation cause a decrease in fluorescence intensity of the red-shifted state as they are formed later in time. This gives rise to the typical excitation dependent fluorescence spectra of CNDs. (d) Jablonski diagram showing multi-emissive states created at different times due to the excited state dynamics of CNDs. As the fluorescence decay and excited state relaxation occurs together, the excited state population gradually decays in the relaxed states or red-shifted species.

are formed later, invariably show lower intensity (Scheme 1c). This can be understood from the excited state energy diagram as shown in Scheme $1 \mathrm{~d}$, where the population intensity of redshifted states gradually drops, as they are formed late, in a time dependent manner (Scheme 1d) [18]. The $\mathrm{pH}$-induced phenol-phenolate equilibrium recently reported by Choudhury et al is very concordant with our twostate model presented here. The model also explains the observation of multiemissive states in various recent studies [24,25] which is often attributed to the core, surface and edge structure without any direct evidence. It should be noted here that this phenomena is most relevant, yet, difficult to identify when the two emissive species have overlapping spectra. This manifests as a broadened steady state spectrum, and gives rise to excitation dependent emission or spectral heterogeneity. In contrast, in this study, the emission maxima of the two species are separated by $\sim 100 \mathrm{~nm}$; as a result, individual Gaussian peaks are easily identifiable (Figure 1a). Hence, though apparently unrelated, this study provides unique evidences for two species emission and their role in excitation dependent emission of CNDs.

\section{Amplified spontaneous emission from CNDs}

Finally, we observed amplified spontaneous emission (ASE), one of the important criteria for lasing, from the protonated state of CNDs at low $\mathrm{pH}$ conditions. The high fluorescence lifetime value and a negligible overlap between absorption and emission spectra only at lower $\mathrm{pH}$, provide a unique condition for ASE to occur in aqueous solution. It is important to note that ASE has already been observed from CNDs (mostly in organic solvents) in some previous studies showing the potential of CNDs in lasing action [26,27]. We used a simple mirror assembly to mimic an optical cavity inside a spectrophotometer. The basic diagram is shown in Supplementary Figure S5. To generate a continuous pulsed light source we used the kinetic mode of fluorescence measurement with $10 \mathrm{~ms}$ interval, $10 \mathrm{~ms}$ integration time and a total measurement time of $30 \mathrm{~m}$. The emission position was fixed at $465 \mathrm{~nm}$ and the $280 \mathrm{~nm}$ light was used for excitation. While a usual constant signal is observed in all control experiments, a steady signal increment was observed using the mirror assembly (Figures 4 and S5 (Supplementary)). This preliminary observation is extremely important as it strongly indicates the potential of this material to be used in future studies on lasing and optical enhancement. Moreover, the tunabilty of lasing action with $\mathrm{pH}$ is a unique and useful feature in our study. As the pulse rate is very slow in this case, population inversion is very unlikely to be the reason of this gain. However, the presence of the mirrors will 
Citation: Syamantak K, Navneet CV, Prashant G, Sanjhal J, Souvik G, et al. (2018) Mechanistic Insight into the Carbon Dots: Protonation induced Photoluminescence. J Material Sci Eng 7: 448. doi: 10.4172/2169-0022.1000448

Page 6 of 6

reflect the emitted light of $465 \mathrm{~nm}$ multiple times, which might cause stimulated emission to happen to some extent. This is one explanation behind the signal increment in kinetic mode of a spectrofluoremeter. Although, future studies are required to clearly understand such phenomena, we present CNDs with a proof of principle of its potential in optoelectronics.

\section{Conclusion}

So in summary, a two-state model of protonated and deprotonated excited states provides new insights into the CNDs photoluminescence. Emission of CNDs shows some resemblance with naphthol and benzoic acid providing some understanding of the possible photoluminescence origin, i.e., the $\mathrm{pH}$ dependence of CNDs photoluminescence originates from the protonation-deprotonation of hydroxyl and carboxyl groups, often in the excited state. It is to be noted here that the state mixing can create more than two emissive states resulting much more complex photoluminescence. Therefore future research is necessary in this direction to get more insight of the photoluminescence process. We also show a proof of principle of tunable ASE from CNDs, which could be useful for future lasing applications.

\section{References}

1. Sun YP, Zhou B, Lin Y, Wang W, Fernando KAS, et al. (2006) Quantum-Sized Carbon Dots for Bright and Colorful Photoluminescence. J Am Chem Soc 128: 7756-7757.

2. Wang L, Zhu SJ, Wang HY, Qu SN, Zhang YL, et al. (2014) Common Origin of Green Luminescence in Carbon Nanodots and Graphene Quantum Dots. ACS Nano 8: 2541-2547.

3. Baker SN, Baker GA (2010) Luminescent Carbon Nanodots: Emergent Nanolights. Angewandte Chem, Int Ed 49: 6726-6744.

4. Nie H, Li M, Li Q, Liang S, Tan Y, et al. (2014) Carbon Dots with Continuously Tunable Full-Color Emission and Their Application in Ratiometric pH Sensing. Chemistry of Materials 26: 3104-3112.

5. Dekaliuk MO, Viagin O, Malyukin YV, Demchenko AP (2014) Fluorescent carbon nanomaterials: "quantum dots" or nanoclusters? Phys Chem Chem Phys 16: 16075-16084

6. Das SK, Liu Y, Yeom S, Kim DY, Richards Cl (2014) Single-Particle Fluorescence Intensity Fluctuations of Carbon Nanodots. Nano Lett 14 620-625.

7. Tong G, Wang J, Wang R, Guo X, He L, et al. (2015) Amorphous carbon dots with high two-photon fluorescence for cellular imaging passivated by hyperbranched poly(amino amine). J Mater Chem B 3: 700-706.

8. Margraf JT, Strauss V, Guldi DM, Clark T (2015) The Electronic Structure of Amorphous Carbon Nanodots. J Phys Chem B 119: 7258-7265.

9. Krysmann MJ, Kelarakis A, Dallas P, Giannelis EP (2012) Formation Mechanism of Carbogenic Nanoparticles with Dual Photoluminescence Emission. J Am Chem Soc 2: 747-750.

10. Lim SY, Shen W, Gao Z (2015) Carbon quantum dots and their applications. Chem Soc Rev 44: 362-381.
11. Kozak O, Sudolská M, Pramanik G, Cígler P, Otyepka M (2016) Photoluminescent Carbon Nanostructures. Chem Mater 28: 4085-4128.

12. Zhao M, Yang F, Xue Y, Xiao D, Guo Y (2014) A Time-Dependent DFT Study of the Absorption and Fluorescence Properties of Graphene Quantum Dots. Chem Phys Chem 15: 950-957.

13. Zheng C, An X, Gong J (2015) Novel pH sensitive N-doped carbon dots with both long fluorescence lifetime and high quantum yield. RSC Advances 5: 32319-32322.

14. Shi L, Yang JH, Zeng HB, Chen YM, Yang SC, et al. (2016) Carbon dots with high fluorescence quantum yield: the fluorescence originates from organic fluorophores. Nanoscale 8: 14374-14378.

15. Konkena B, Vasudevan S (2013) Spectral Migration of Fluorescence in Graphene Oxide Aqueous Dispersions: Evidence for Excited-State Proton Transfer. J Phys Chem Lett 5: 1-7.

16. Dutta Choudhury S, Chethodil JM, Gharat PM, Pal H (2017) pH-Elicited Luminescence Functionalities of Carbon Dots: Mechanistic Insights. J Phys Chem Lett 8: 1389-1395.

17. Gupta A, Verma NC, Khan S, Tiwari S, Chaudhary A, et al. (2016) Paper strip based and live cell ultrasensitive lead sensor using carbon dots synthesized from biological media. Sensors and Actuators B: Chemical 232: 107-114.

18. Khan S, Gupta A, Verma NC, Nandi CK (2015) Time-Resolved Emission Reveals Ensemble of Emissive States as the Origin of Multicolor Fluorescence in Carbon Dots. Nano Lett 15: 8300-8305.

19. Koti ASR, Krishna MMG, Periasamy N (2001) Time-Resolved Area-Normalized Emission Spectroscopy (TRANES): A Novel Method for Confirming Emission from Two Excited States. J Phys Chem A 105: 1767-1771.

20. Koti A, Krishna M, Periasamy N (2001) Time-resolved area-normalized emission spectroscopy (TRANES): A novel method for confirming emission from two excited states. J Phys Chem A 105: 1767-1771

21. Koti AS, Periasamy N (2001) TRANES analysis of the fluorescence of nile red in organized molecular assemblies confirms emission from two species. J Chem Sci 113: 157-163.

22. Laws WR, Brand L (1979) Analysis of two-state excited-state reactions. The fluorescence decay of 2-naphthol. J Phys Chem 83: 795-802.

23. Marciniak B, Kozubek H, Paszyc S (1992) Estimation of $\mathrm{pKa}^{*}$ in the first excited singlet state. A physical chemistry experiment that explores acid-base properties in the excited state. J Chem Educ 69: 247.

24. Dhenadhayalan N, Lin KC, Suresh R, Ramamurthy $P$ (2016) Unravelling the Multiple Emissive States in Citric-Acid-Derived Carbon Dots. J Phys Chem C 120: $1252-1261$

25. Sharma A, Gadly T, Neogy S, Ghosh SK, Kumbhakar M (2017) Molecular Origin and Self-Assembly of Fluorescent Carbon Nanodots in Polar Solvents. J Phys Chem Lett 8: 1044-1052.

26. Qu S, Liu X, Guo X, Chu M, Zhang L, et al. (2014) Amplified spontaneous green emission and lasing emission from carbon nanoparticles. Adv Funct Mater 24 2689-2695.

27. Zhang WF, Tang LB, Yu SF, Lau SP (2012) Observation of white-light amplified spontaneous emission from carbon nanodots under laser excitation. Opt Mater Express 2: 490-495. 